\title{
Appropriate timing for hypothermic machine perfusion to preserve livers donated after circulatory death
}

\author{
XIAOYAN HU ${ }^{1}$, WEI WANG ${ }^{1}$, CHENG ZENG $^{1}$, WEIYANG HE ${ }^{1}$, \\ ZIBIAO ZHONG $^{1}$, ZHONGZHONG LIU $^{1}$, YANFENG WANG ${ }^{1}$ and QIFA YE $^{1,2}$ \\ ${ }^{1}$ Zhongnan Hospital of Wuhan University, Institute of Hepatobiliary Diseases of Wuhan University, \\ Transplant Center of Wuhan University, Hubei Key Laboratory of Medical Technology on Transplantation, \\ Wuhan, Hubei $430071 ;{ }^{2}$ The 3rd Xiangya Hospital of Central South University, Research Center of \\ National Health Ministry on Transplantation Medicine Engineering and Technology, Changsha, Hunan 410013, P.R. China
}

Received September 5, 2019; Accepted May 28, 2020

DOI: $10.3892 / \mathrm{mmr} .2020 .11257$

\begin{abstract}
Hypothermic machine perfusion (HMP) is a method that can be more effective in preserving donor organs compared with cold storage (CS). However, the optimal duration and the exact mechanisms of the protevtive effects of HMP remain unknow. The present study aimed to investigate the adequate perfusion time and mechanisms underlying HMP to protect livers donated after circulatory death (DCD). After circulatory death, adult male Sprague-Dawley rat livers were subjected to 30 min of warm ischemia (WI) and were subsequently preserved by HMP or CS. To determine the optimal perfusion time, liver tissues were analyzed at 0 , $1,3,5,12$ and $24 \mathrm{~h}$ post-preservation to evaluate injury and assess the expression of relevant proteins. WI livers were preserved by HMP or CS for $3 \mathrm{~h}$, and liver viability was evaluated by normothermic reperfusion (NR). During NR, oxygen consumption, bile production and the activities of hepatic enzymes in the perfusate were assessed. Following $2 \mathrm{~h}$ of NR, levels of inflammation and oxidative stress were determined in the livers and perfusate. HMP for $3 \mathrm{~h}$ resulted in the highest expression of myocyte enhancer factor $2 \mathrm{C}$ (MEF2C) and kruppel-like factor 2 (KLF2) and the lowest expression of NF- $\mathrm{KB}$ p65, tumor necrosis factor (TNF)- $\alpha$ and interleukin (IL)-1 $\beta$ among the different timepoints, which
\end{abstract}

Correspondence to: Professor Yanfeng Wang or Professor Qifa Ye, Zhongnan Hospital of Wuhan University, Institute of Hepatobiliary Diseases of Wuhan University, Transplant Center of Wuhan University, Hubei Key Laboratory of Medical Technology on Transplantation, 169 Donghu Road, Wuhan, Hubei 430071, P.R. China

E-mail: yanfengwang@whu.edu.cn

E-mail:yqf_china@163.com

Key words: donated after circulatory death, hypothermic machine perfusion, cold storage, normothermic reperfusion, myocyte enhancer factor $2 \mathrm{C}$, kruppel-like factor $2, \mathrm{NF}-\mathrm{\kappa B}$ p 65 , endothelial nitric oxide synthase indicated that $3 \mathrm{~h}$ may be the optimal time for HMP induction of the KLF2-dependent signaling pathway. Compared with CS-preserved livers, HMP-preserved livers displayed significantly higher oxygen consumption, lower hepatic enzyme levels in the perfusate following NR. Following HMP preservation, the expression levels of MEF2C, KLF2, endothelial nitric oxide synthase and nitric oxide were increased, whereas the expression levels of NF- $\mathrm{BB}$ p65, IL- $1 \beta$ and TNF- $\alpha$ were decreased compared with CS preservation. The results indicated that $3 \mathrm{~h}$ may be the optimal time for HMP to protect DCD rat livers. Furthermore, HMP may significantly reduce liver inflammation and oxidative stress injury by mediating the KLF2/NF-kB/eNOS-dependent signaling pathway.

\section{Introduction}

Liver transplantation is the most effective therapeutic strategies for end-stage liver diseases; however, the ever-increasing shortage of donor organs limits the development of liver transplantation and leads to the use of livers from expanded criteria donors (ECDs) (1). As a result, marginal organs that are more susceptible to the harmful effects of ischemia-reperfusion injury (IRI) are frequently used for transplantation (2). IRI is a complicated pathophysiological process that is difficult to avoid during liver transplantation. Multiple mechanisms contribute to the process of IRI, of which inflammation and oxidative stress display major roles $(3,4)$. After transplantation, patients who receive marginal organs are more likely to develop early allograft dysfunction, liver rejection and biliary complications, and thus have a higher risk of unfavorable short- and long-term outcomes (5). Therefore, the effective maintenance and optimization of the quality of organs donated after circulatory death (DCD) are urgent problems in the field of organ transplantation.

In order to attenuate IRI, the organ preservation method of HMP has received increasing interest worldwide $(6,7)$. The first clinical study on liver HMP confirmed its effectiveness for DCD and ECD organs, with HMP displaying improved results compared with cold storage (CS) (8). Previous studies investigating the application of HMP for discarded human livers verified the advantages of the technique $(7,9)$. 
Furthermore, the protective effects of HMP in DCD organs has been reported. In a previous study, HMP was employed for 1-7 $\mathrm{h}$ and displayed beneficial outcomes for all durations (10). However, the optimal duration of HMP to reduce liver injury and maintain optimal liver viability, as well as the exact molecular mechanisms underlying HMP-mediated protection of DCD livers, have not been previously reported.

Lüer et al (11) reported that HMP can upregulate kruppel-like factor 2 (KLF2) expression in livers. KLF2 is a transcriptional regulator that has a zinc-finger structure and is highly expressed in the lungs and vascular endothelium. KLF2 expression can be induced by laminar sheer stress, resulting in atheroprotective, anticoagulant and anti-inflammatory effects $(12,13)$. Several studies have demonstrated that KLF2 can inhibit the transcriptional activity of NF- $\mathrm{KB}$, which controls the transcription and expression of various cytokines and adhesion molecules involved in inflammation and the immune response. For example, NF- $\kappa \mathrm{B}$ can reduce the expression of tumor necrosis factor (TNF)- $\alpha$ and interleukin (IL)-1 $\beta$ to attenuate inflammation (14-20). Wang et al (21) demonstrated that flow-stimulated phosphorylation and nuclear export of histone deacetylase 5 (HDAC5) results in the dissociation of HDAC5 and myocyte enhancer factor 2C (MEF2C), which enhances the transcriptional activity of MEF2C and induces the expression of KLF2 and endothelial nitric oxide synthase (eNOS), increasing nitric oxide (NO) levels in human umbilical vein endothelial cells (ECs) exposed to laminar flow. eNOS is a $\mathrm{Ca}^{2+}$, NADPH-, flavin- and biopterin-dependent enzyme that can constitutively produce NO in various cells. The activity of eNOS is tightly regulated by different mechanisms, of which phosphorylation at specific amino acids can lead to activation or inhibition of eNOS activity depending on the localization in the protein sequence $(22,23)$. Among the numerous phosphorylation sites, Ser1177 is rapidly phosphorylated after the application of fluid shear stress, which results in eNOS activation to sustain moderate NO generation (24). NO displays important biological functions, including vasodilatation, scavenging of superoxide, inhibition of platelet aggregation, and reduction of proliferation and inflammation (22-25). However, only a limited number of studies have investigated the anti-inflammatory and antioxidant effects of the KLF2/NF-kB/eNOS/NO signaling pathway during organ transplantation.

Although HMP has been widely researched, the optimal duration of HMP to effectively attenuate IRI has not been previously reported and the molecular mechanisms underlying the protective effects of HMP are not completely understood. The present study aimed to investigate the optimal duration of liver HMP by comparing anti-inflammatory and antioxidant activities during IRI. In addition, whether the protective effects of HMP were exerted via the KLF2/NF- $\kappa \mathrm{B} / \mathrm{eNOS} / \mathrm{NO}$ signaling was investigated.

\section{Materials and methods}

Animals. A total of 42 adult male Sprague Dawley (SD) rats (age, 8-10 weeks; weight, 250-300 g) were purchased from the Experimental Animal Culture Center of the Hubei Center for Disease Control. All animals received standard care and were housed under standard laboratory conditions (temperature, $25 \pm 2^{\circ} \mathrm{C}$; relative humidity, $55 \pm 5 \%$; a $12 \mathrm{~h}$ light/dark cycle) in Zhongnan Hospital's Animal Experiment Center of Wuhan University. Animals had free access to food and water. The present study was approved by the Ethical Committee of Wuhan University and carried out in accordance with the Experimental Animal Management Ordinance (National Science and Technology Committee of China) and the Guide for the Care and Use of Laboratory Animals (National Institutes of Health) (26).

Establishment of the rat model of DCD. Rats were anesthetized by the intraperitoneal injection of pentobarbital sodium $(50 \mathrm{mg} / \mathrm{kg})$. An abdominal longitudinal incision was made, and the hepatic artery, portal vein, supra- and infrahepatic inferior vena cava, bile duct and peripheral ligaments were freed to fully expose the liver. An epidural guiding conduit was inserted (Jiangsu Changfeng Medical Industry Co., Ltd.) into the bile duct. After ligation of the left phrenic vein, the diaphragm was cut to induce bilateral pneumothorax and cardiac arrest. The period of WI started from the point of cardiac arrest (27). After 30 min of WI, systemic heparinization was implemented by the injection of $2 \mathrm{ml}$ Ringer (Chimin Health Management Co., Ltd.; www.chimin.cn) and $100 \mathrm{IU}$ heparin (Hepatunn; http://www.hepatunn.com/) via the right iliac vein (28). Subsequently, the hepatic artery was ligated and the liver was flushed in situ with $20 \mathrm{ml} \mathrm{0-4}{ }^{\circ} \mathrm{C}$ Histidine Tryptophan Ketoglutarate (HTK) solution (Dr Franz Koehler Chemie $\mathrm{GmbH}$ ) via the portal vein intubation [homemade pressure equalizer (PE) tubes; outer diameter, $2.1 \mathrm{~mm}$; inner diameter, $1.8 \mathrm{~mm}$ ]. To collect the hepatic effluent, the suprahepatic inferior vena cava was intubated using a PE catheter (inner diameter, $3 \mathrm{~mm}$ ) (29).

Preservation of DCD livers. SD rats were randomly divided into the HMP group and the CS group. In the HMP group $(\mathrm{n}=18)$, the livers were obtained after $30 \mathrm{~min}$ of $\mathrm{WI}$ and $0-4^{\circ} \mathrm{C}$ HTK solution $(150 \mathrm{ml})$ was perfused via the portal vein for $0,1,3,5,12$ or $24 \mathrm{~h}$ at a rate of $0.5 \mathrm{ml} / \mathrm{g} / \mathrm{min}(29)$. At each time point, three livers were harvested. In the CS group $(\mathrm{n}=18)$, after $30 \mathrm{~min}$ of $\mathrm{WI}$, the livers were maintained in CS $\left(0-4^{\circ} \mathrm{C}\right)$ in HTK solution $(150 \mathrm{ml})$ for $0,1,3,5,12$ or $24 \mathrm{~h}$. At each time point, three livers were harvested. The WI group consisted of livers harvested at $0 \mathrm{~h}$ in both groups.

Evaluation of preservation effects. SD rats were randomly divided into the HMP + normothermic reperfusion (NR) group and the CS + NR group. In the HMP + NR group ( $n=3)$, HMP was performed for $3 \mathrm{~h}$ followed by NR for $2 \mathrm{~h}$ using the isolated perfused rat liver (IPRL) system. In the CS + NR group ( $\mathrm{n}=3$ ), livers were maintained in CS for $3 \mathrm{~h}$ followed by $\mathrm{NR}$ for $2 \mathrm{~h}$ using the IPRL system.

Liver perfusion system. A thermostat water bath (Jiaxingjunsi Electronics Co., Ltd.) containing a liver perfusion box was used. The temperature of the bath was controlled by heated water around the box or by an ice-water mixture inside the box. The temperature of the liver perfusion box was monitored using a temperature sensor (Xinghe Electronics Co., Ltd.). A peristaltic pump (Longerpump Technologies Inc.; https://www.longerpump.com/), a hollow-fiber membrane 
oxygenator (Dongguan Kewei Medical Instrument Co., Ltd.) and a flow meter (Hehua Mechanical and Electric Corporation; https://hqn640901.d17.cc/) were connected to complete the perfusion system. The portal vein cannula was connected to the baroreceptor of a BL-420F Biological Functional System (Chengdu Taimeng Science and Technology Co., Ltd.; http://www.tme.com.cn/) to measure the portal perfusion pressure.

IPRL system. After HMP or CS, livers were rewarmed for 20 min to simulate the period of rewarming during transplantation. Subsequently, the liver was connected to the IPRL system, which consists of a liver perfusion system with an oxygen supply. The temperature of the water bath was set to $40^{\circ} \mathrm{C}$ to maintain the temperature of the liver container at $36.5 \pm 0.5^{\circ} \mathrm{C}$. Krebs-Henseleit buffer (Macgene; http://www.macgene.com/) with 4\% dextran (Xian Wanlong Pharmaceutical Co., Ltd.; http://www.xawanlong.com/) was used for reperfusion (30). The perfusate was oxygenated with $95 \% \mathrm{O}_{2}+5 \% \mathrm{CO}_{2}$ gas to maintain $>500 \mathrm{mmHg}$ oxygen pressure. During the $2 \mathrm{~h}$ perfusion, the portal vein perfusion pressure was maintained at $10.3 \mathrm{mmHg}(10,31)$.

Biochemical examination. During the $2 \mathrm{~h}$ reperfusion, alanine transaminase (ALT; cat. no. C009; Nanjing Jiancheng Bioengineering Institute), aspartate transaminase (AST; cat. no. C010; Nanjing Jiancheng Bioengineering Institute) and lactate dehydrogenase (LDH; cat. no. A020; Nanjing Jiancheng Bioengineering Institute) levels were measured in the hepatic effluent that was collected from the suprahepatic vena cava every 60 min using commercial standard kits, according to the manufacturer's protocol.

Oxygen consumption. To assess the metabolic activity of the livers, hepatic oxygen consumption was analyzed every 60 min during the $2 \mathrm{~h}$ reperfusion using an i-STAT pH-blood gas analyzer (Abbott Point of Care, Inc.). Oxygen consumption ( $\mu \mathrm{mol} / \mathrm{min} / \mathrm{g}$ liver) was calculated according to the following formula: $\left(\mathrm{C}_{\mathrm{in}}-\mathrm{C}_{\mathrm{out}}\right)$ / portal flow $(\mathrm{ml} / \mathrm{min}) /$ liver weight $(\mathrm{g})$, where $\mathrm{C}_{\text {in }}$ and $\mathrm{C}_{\text {out }}$ represent the oxygen concentration of the liver inflow and outflow, respectively (31).

Bile production. Bile was collected every $60 \mathrm{~min}$ via the epidural guiding tube that was positioned in the bile duct. Bile flow is expressed as $\mu \mathrm{l} / \mathrm{h} / \mathrm{g}$ liver, as the density of bile is almost equal to that of water (10).

Liver histology. Liver samples were stored in $10 \%$ formaldehyde at room temperature for 1-5 days, embedded in paraffin, and cut into $3-\mu \mathrm{m}$ sections. Subsequently, sections were stained with hematoxylin and eosin (HE) at room temperature (hematoxylin staining for 5-10 $\mathrm{min}$ and eosin staining for 1-3 min). The severity of hepatic injury was assessed by pathologists who were blinded to the experiment using a light microscope at x200 magnification according to the classification described by Suzuki et al (32). Breifly, sinusoidal congestion, hepatocyte necrosis and ballooning degeneration were graded from 0 to 4 , where 0 indicated no necrosis, congestion or ballooning, and 4 indicated severe congestion, ballooning degeneration or hepatocyte necrosis $(>60 \%)$.
Western blotting. Total protein was extracted from liver tissues using RIPA lysis buffer (Beyotime Institue of Biotechnology; cat. no. P0013B) containing protease inhibitor and quantified by the bicinchoninic acid method. Protein (40 $\mu \mathrm{g} / \mathrm{lane})$ was separated via 10\% SDS-PAGE and transferred onto PVDF membranes, which were blocked with $5 \%$ skim milk (cat. no. G5002; Wuhan Servicebio Technology Co., Ltd.) at room temperature for $2 \mathrm{~h}$. Subsequently, the membranes were incubated overnight at $4^{\circ} \mathrm{C}$ with the following primary antibodies: anti-MEF2C (rabbit; 1:500; cat. no. 10056-1-AP; ProteinTech Group, Inc.), anti-KLF2 (rabbit; 1:400; cat. no. bs-2772R; Beijing Biosynthesis Biotechnology Co., Ltd.), anti-NF-кB p65 (rabbit; 1:400; cat. no. bs-20355R; Beijing Biosynthesis Biotechnology Co., Ltd.), anti-eNOS (rabbit; 1:1,000; cat. no. 20116-1-AP; ProteinTech Group, Inc.), anti-phosphorylated eNOS at Ser1177 (rabbit; 1:1,000; cat. no. 9571, Cell Signaling Technology, Inc.) and anti- $\beta$-actin (rabbit; $1: 1500$; cat. no. bs-0061R; Beijing Biosynthesis Biotechnology Co., Ltd.). After washing with TBST (Tris, $2.42 \mathrm{~g}$; NaCl, $8 \mathrm{~g}$; Tween, $1 \mathrm{ml}$; Distilled Water, $1,000 \mathrm{ml}$ ), the membranes were incubated with horseradish peroxidase-conjugated goat anti-rabbit IgG (H+L) antibodies (1:3,000; cat. no. G1213; Wuhan Servicebio Technology Co., Ltd.) at room temperature for $2 \mathrm{~h}$. Protein bands were visualized using chemiluminescence ECL reagent (cat. no. AR1172; Boster Biological Technology). Protein expression was quantified using ImageJ software (version 1.42q; National Institutes of Health) with $\beta$-actin as the loading control.

ELISA. TNF- $\alpha$ (cat. no. ERC102a; QuantiCyto, http://www. neobioscience.net/pro_view-7421.html) and IL-1 $\beta$ (cat. no. ERC007; QuantiCyto; http://www.neobioscience.net/ pro_view-7346.html) expression levels in liver tissues and NO levels (cat. no. A012; Nanjing Jiancheng Bioengineering Institute) in the perfusate were measured using ELISA kits.

Statistical analysis. Statistical analyses were performed using SPSS software (version 17.0; SPSS, Inc.). Data are presented as the mean \pm standard error. The normality of each results was analyzed by homogeneity of variance analysis. Differences between two groups were analyzed using the unpaired Student's t-test. $\mathrm{P}<0.05$ was considered to indicate a statistically significant difference.

\section{Results}

HMP attenuates WI-induced pathological liver injury. First, hepatic pathological injury was assessed (Fig. 1). Injury was quantified using a previously validated liver pathological damage scoring system (32), where a higher score indicated greater damage. After 30 min of WI, livers displayed significant sinusoidal congestion, hepatocyte edema, anoxic vacuoles, dot necrosis and high numbers of lymphocytes. In the livers of the CS group, severe hepatocellular swelling/necrosis, sinusoidal congestion and inflammatory cell infiltration was observed. The livers of the HMP group displayed less damage compared with the CS group (Fig. 1A). Moreover, the HMP group displayed a significantly lower pathology score compared with the CS group at 3, 5, 12 and $24 \mathrm{~h}(\mathrm{P}<0.05)$, with the lowest score recorded at the $3 \mathrm{~h}$ timepoint. At the $1 \mathrm{~h}$ timepoint, the 
A

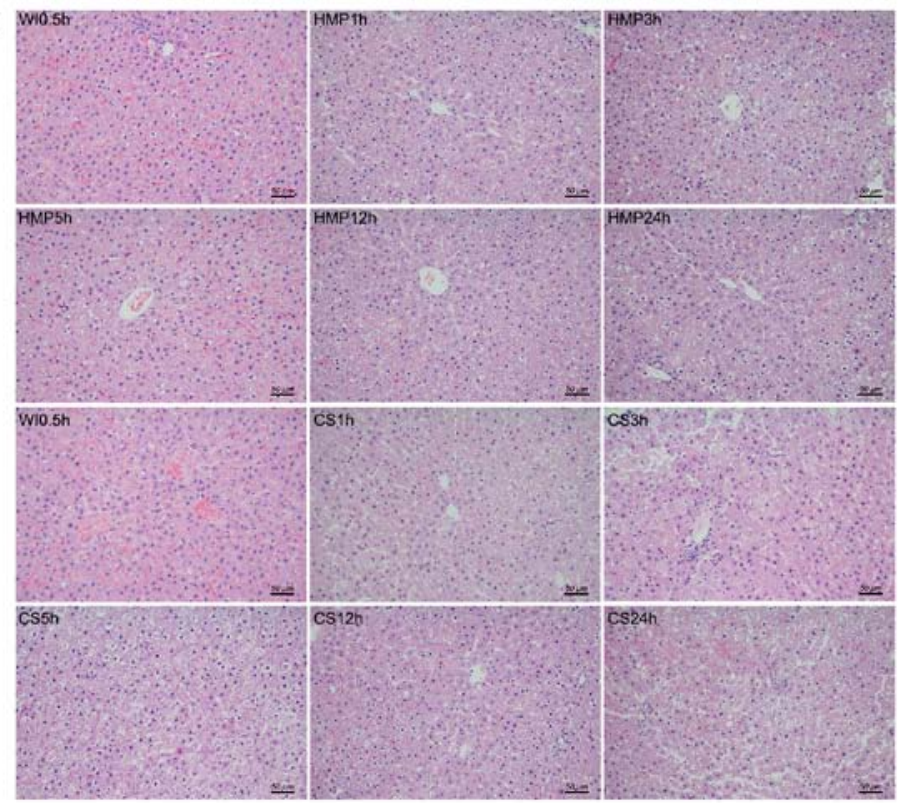

B

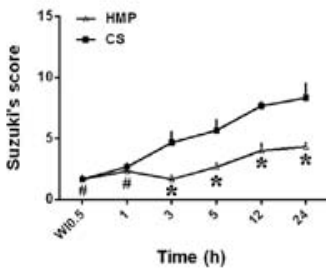

Figure 1. HMP attenuates ischemia-induced hepatic pathological damage. (A) Hepatic tissue sections were stained with hematoxylin and eosin (magnification, x200). (B) The degree of damage was graded using Suzuki's scoring system. ${ }^{*} \mathrm{P}<0.05$ vs. CS; ${ }^{\text {P }}>0.05$ vs. CS. HMP, hypothermic machine perfusion; CS, cold storage; WI, warm ischemia.

difference in pathology score was not significant between the HMP and CS groups ( $\mathrm{P}>0.05$; Fig. 1B). The results suggested that HMP preserved DCD livers more effectively compared with CS.

$H M P$ increases MEF2C and KLF2 expression and decreases $N F-\kappa B$ p 65, TNF- $\alpha$ and IL-1 $\beta$ expression during the ischemic phase. Subsequently, the protein expression levels of MEF2C, KLF2 and NF- $\kappa$ B p65 were assessed by western blotting to determine the effects of HMP on hepatic inflammation (Fig. 2). MEF2C and KLF2 expression levels were significantly increased in the HMP group compared with the CS group at 3, 5, 12 and $24 \mathrm{~h}(\mathrm{P}<0.05$; Fig. $2 \mathrm{~B}$ and $\mathrm{C})$. By contrast, the expression levels of NF- $\mathrm{B}$ p 65 were significantly lower in the HMP group compared with the CS group at 3, 5, 12 and $24 \mathrm{~h}(\mathrm{P}<0.05$; Fig. 2D). TNF- $\alpha$ and IL-1 $\beta$ are downstream

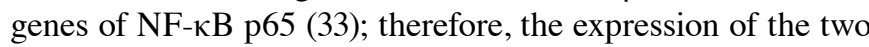
inflammatory cytokines was measured by ELISA to assess the degree of NF- $\mathrm{B}$ p 65 -induced inflammation. TNF- $\alpha$ and IL- $1 \beta$ levels were significantly lower in the HMP group compared with the CS group at 3, 5, 12 and $24 \mathrm{~h}(\mathrm{P}<0.05$; Fig. $2 \mathrm{E}$ and $\mathrm{F})$. For all genes, the most notable difference between the HMP group and the CS group was observed at the $3 \mathrm{~h}$ timepoint. However, the expression levels of the examined genes were not significantly different between the HMP and CS groups at the $1 \mathrm{~h}$ timepoint $(\mathrm{P}>0.05)$.

HMP alleviates reperfusion-induced hepatocellular injury. The protective effects of HMP were further assessed using the IPRL system, which can imitate the physical environment. The release of ALT, AST and LDH during reperfusion was measured. ALT, AST and LDH concentrations sharply increased following reperfusion in both the HMP and CS groups (Fig. 3). However, enzyme levels were significantly lower in the HMP group compared with the CS group at
60 and $120 \mathrm{~min}(\mathrm{P}<0.05$; Fig. 3A-C). Livers in the HMP group displayed significantly higher rates of oxygen consumption compared with the CS group at 0,60 and $120 \mathrm{~min}(\mathrm{P}<0.05$; Fig. 3D). In addition, bile production was slightly higher in the HMP group compared with the CS group, but the difference was not statistically significant at $120 \mathrm{~min}$ ( $\mathrm{P}>0.05$; Fig. 3E).

HMP increases MEF $2 C$ and KLF2 expression and decreases $N F-\kappa B$ p 65, IL-1 $\beta$ and TNF- $\alpha$ expression during the reperfusion phase. Based on the observation that HMP for $3 \mathrm{~h}$ increased MEF2C and KLF2 expression and decreased NF- $\kappa \mathrm{B}$ p65, TNF- $\alpha$ and IL-1 $\beta$ expression (Fig. 2), the protective mechanisms underlying HMP were further investigated by evaluating protein expression after NR. MEF2C and KLF2 expression levels were significantly increased in livers of the $\mathrm{HMP}+\mathrm{NR}$ group compared with the $\mathrm{CS}+\mathrm{NR}$ group $(\mathrm{P}<0.05$; Fig. 4A-C). By contrast, NF- $\kappa$ B p65, TNF- $\alpha$ and IL- $1 \beta$ expression levels were significantly decreased in the HMP + NR group compared with the CS + NR group ( $\mathrm{P}<0.05$; Fig. 4D-F). The results indicated that HMP may attenuate IRI-induced hepatic inflammation.

HMP increases eNOS and NO levels during the reperfusion phase. Finally, whether HMP could reduce IRI-associated oxidative stress was assessed by evaluating the expression of eNOS and p-eNOS at Ser1177. In livers preserved by HMP, significantly higher levels of eNOS and p-eNOS were observed compared with livers preserved by CS, and the ratio of $\mathrm{p}$-eNOS/eNOS was also significantly increased $(\mathrm{P}<0.05$; Fig. 5A-D). eNOS constitutively produces NO; therefore, NO levels in the perfusate after $2 \mathrm{~h}$ of NR were also measured by ELISA. NO levels were significantly higher in the HMP group compared with the CS group $(\mathrm{P}<0.05$; Fig. 5E). Therefore, the results indicated that HMP reduced hepatic oxidative stress during IRI. 
A

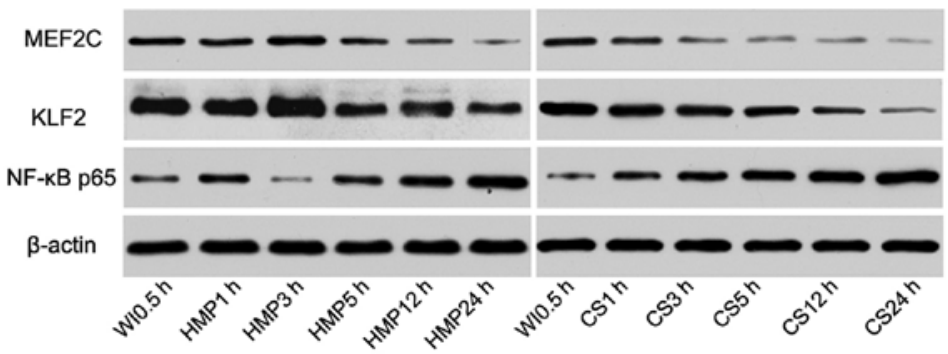

B

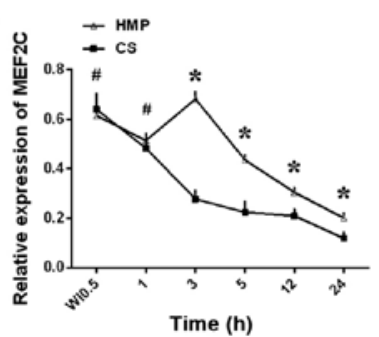

C

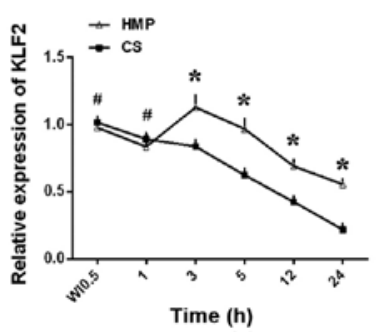

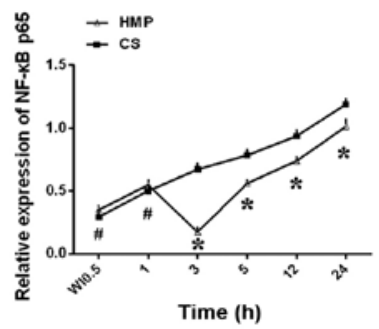

E

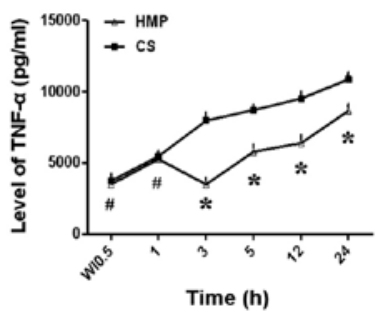

$\mathbf{F}$

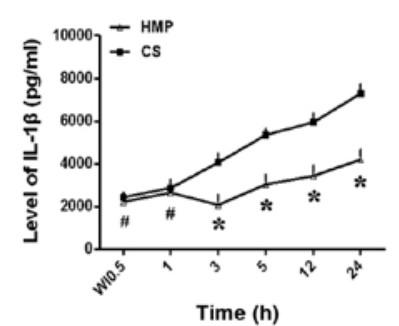

Figure 2. Expression levels of MEF2C, KLF2, NF- $\mathrm{kB}$ p65, TNF- $\alpha$ and IL-1 $\beta$ in liver tissues. Protein expression levels were (A) determined by western blotting and semi-quantified for (B) MEF2C, (C) KLF2 and (D) NF- $\kappa$ B p65. (E) TNF- $\alpha$ and (F) IL-1 $\beta$ expression levels were assessed by ELISA. "P<0.05 vs. CS ${ }^{\#} \mathrm{P}>0.05$ vs. CS. MEF2C, myocyte enhancer factor 2; KLF2, kruppel-like factor 2; TNF- $\alpha$, tumor necrosis factor- $\alpha$; IL-1 $\beta$, interleukin- $1 \beta ;$; , cold storage; HMP, hypothermic machine perfusion; WI, warm ischemia.
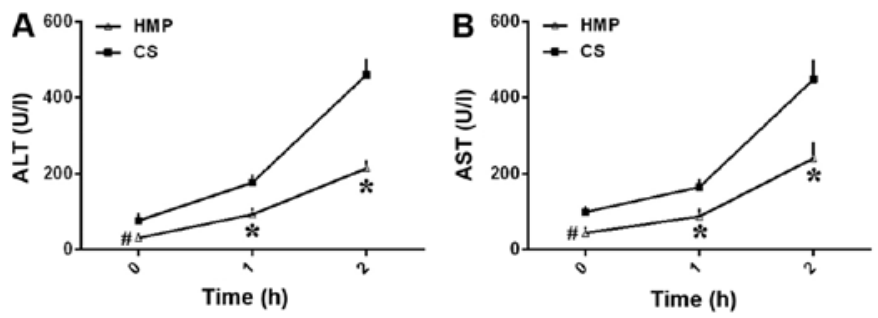

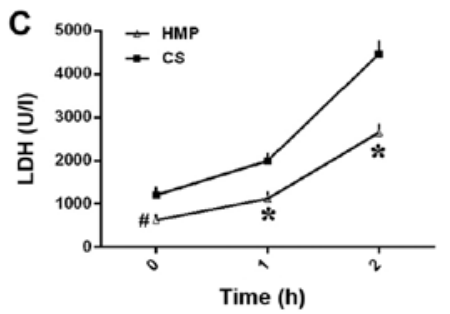

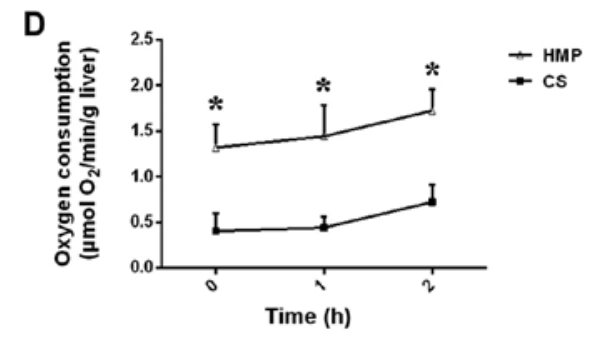

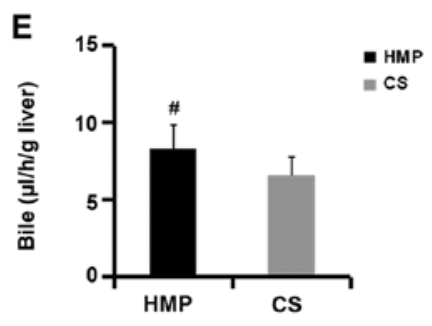

Figure 3. Evaluation of hepatocellular injury after NR. Release of (A) ALT, (B) AST and (C) LDH during NR after 3 h of HMP or CS. (D) Oxygen consumption and (E) bile production during NR after 3 h of HMP or CS. "P $<0.05$ vs. CS; " $\mathrm{P}>0.05$ vs. CS. NR, normothermic reperfusion; ALT, alanine transaminase; AST, aspartate transaminase; LDH, lactate dehydrogenase; HMP, hypothermic machine perfusion; CS, cold storage.

\section{Discussion}

The increased demand for organs has led to the use of ECD and DCD organs, which often require longer periods of WI or cold ischemia, resulting in a higher risk of serious injury and unfavorable outcomes $(2,6)$. Liver IRI is a complex pathophysiological response that cannot be fully avoided during liver transplantation. Oxidative stress and inflammatory responses are the main mechanisms underlying liver damage during liver transplantation $(3,4)$. Donor organs with impaired 

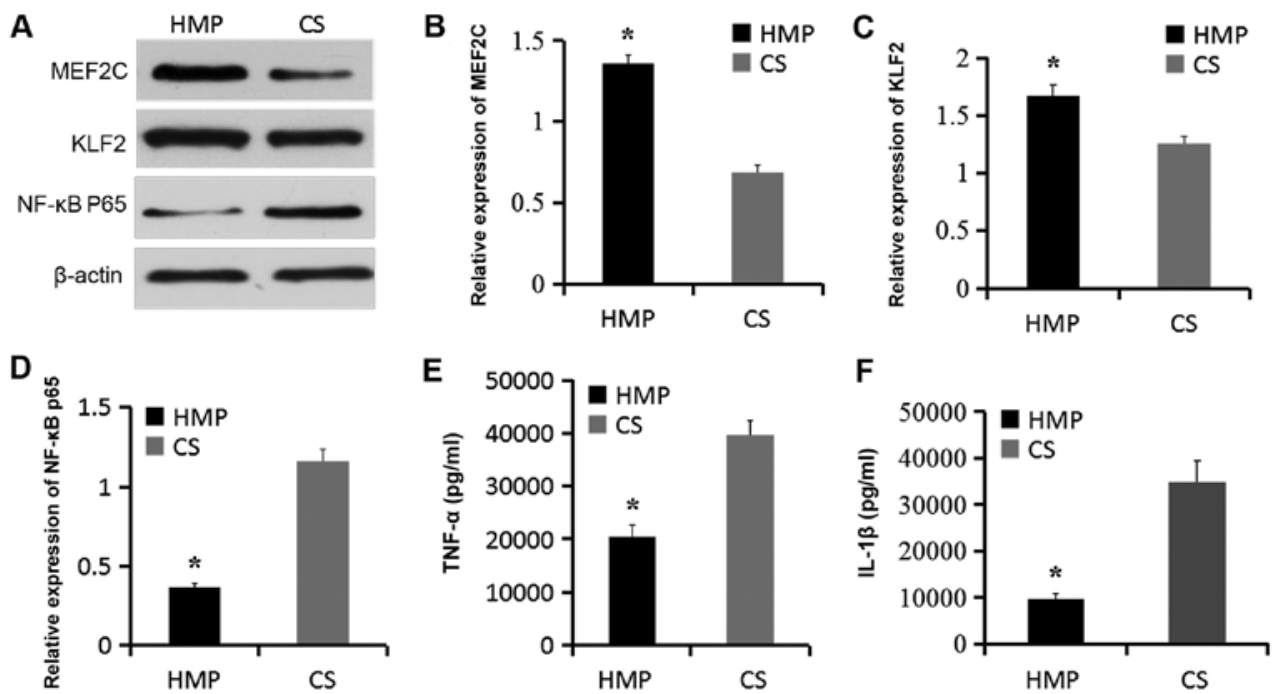

Figure 4. Expression levels of MEF2C, KLF2, NF- $\mathrm{BB}$ p65, TNF- $\alpha$ and IL-1 $\beta$ after NR. Protein expression levels were (A) determined by western blotting and semi-quantified for (B) MEF2C, (C) KLF2 and (D) NF-kB p65. Expression levels of the inflammatory cytokines (E) TNF- $\alpha$ and (F) IL-1 $\beta$ were measured by ELISA. "P<0.05 vs. CS. MEF2C, myocyte enhancer factor 2; KLF2, kruppel-like factor 2; TNF- $\alpha$, tumor necrosis factor- $\alpha$; IL- $1 \beta$, interleukin-1 $\beta$; NR, normothermic reperfusion; CS, cold storage; HMP, hypothermic machine perfusion.

A

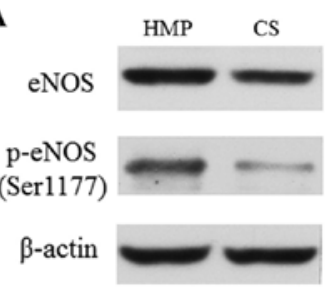

B

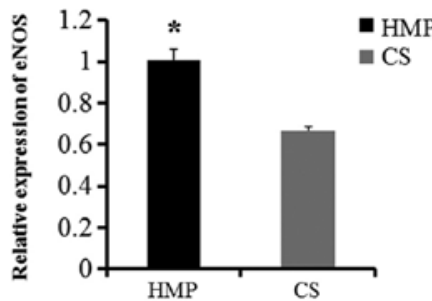

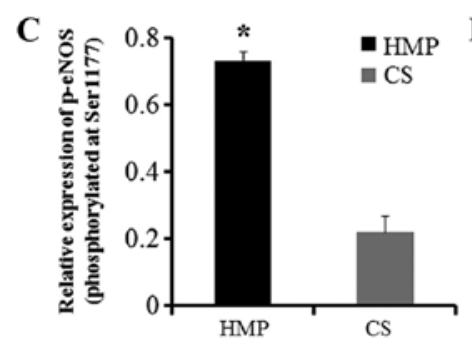
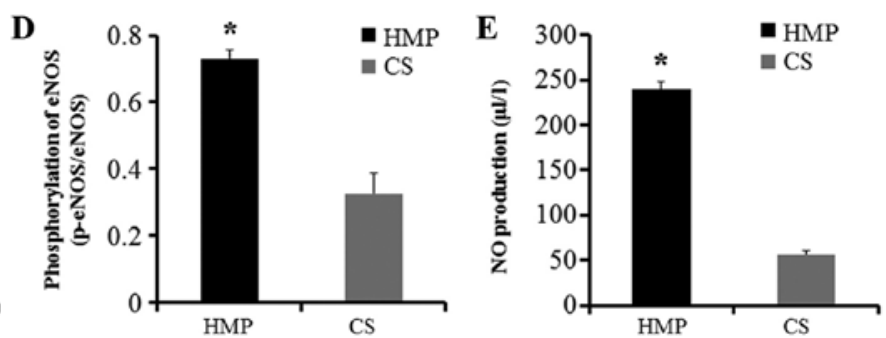

Figure 5. Expression levels of eNOS, p-eNOS and NO after NR. Protein expression levels were (A) determined by western blotting and semi-quantified for (B) eNOS and (C) p-eNOS. (D) The ratio of total eNOS/p-eNOS. (E) NO levels in the perfusate. " $\mathrm{P}<0.05$ vs. CS. eNOS, endothelial nitric oxide synthase; $\mathrm{p}$, phosphorylated; NO, nitric oxide; NR, normothermic reperfusion; CS, cold storage; HMP, hypothermic machine perfusion.

function severely threaten the short- and long-term prognosis of the patient receiving the liver transplantation $(1,2)$; therefore, identifying strategies that focus on reducing liver IRI and preserving organ quality are of high importance.

At present, HMP and CS are the two most commonly used strategies for organ preservation (34). Numerous studies have demonstrated that HMP preserves organs more effectively compared with CS (7-10). HMP, which can cause vascular shear stress by pumping perfusate, allows for organ optimization and offers a platform for viability assessment, organ repair and resuscitation $(35,36)$. Despite the extensive studies on HMP, the optimal perfusion time has not been reported and the molecular mechanisms underlying HMP are not completely understood. In the present study, a well-established animal model was used to generate DCD livers, which were then preserved by HMP or CS. Liver quality was assessed at six different time points $(0,1,3,5,12$ and $24 \mathrm{~h})$. The results suggsted that HMP preserved DCD livers more effectively compared with CS, which was consistent with our previous studies $(6,10,13)$. In addition, morphological alterations to the liver tissues indicated that the protective effect of HMP was most notable at the $3 \mathrm{~h}$ timepoint.

Vascularshearstress serves an important rolein theregulation of vascular function, while laminar shear stress mechanically stimulates vascular endothelium and induces the expression of flow-dependent vascular protective genes $(12,21,37)$. Moreover, laminar flow can activate a number of distinct signaling pathways in ECs to modulate transcription factor activity (37), with the MEF2 transcription family being an example. MEF2 proteins are members of the MCM1 agamous-deficiens-serum 
response factor family of transcription factors, which bind to AT-rich sequences. There are four isoforms of MEF2: MEF2A, MEF2B, MEF2C and MEF2D (38). MEF2C displays a key role during $\mathrm{EC}$ angiogenesis (39), and $\mathrm{Xu}$ et al (40) reported that MEF2C suppresses EC inflammation by regulating $\mathrm{NF}-\kappa \mathrm{B}$ and KLF2 expression. The most extensively studied in vitro targets of MEF2 in ECs are KLF2 and KLF4, which regulate antithrombotic and anti-inflammatory transcriptional signaling pathways (41). Parmar et al (42) demonstrated that overexpression of a dominant-negative MEF2 variant prevents flow-mediated induction of KLF2 expression in ECs. As an important flow-regulated molecule, KLF2 has greatly advanced the understanding of the molecular mechanisms underlying vascular homeostasis $(12,15,37)$. KLF2 interacts with $\mathrm{NF}-\kappa \mathrm{B}$ during the inflammatory immune response, which activates $\mathrm{NF}-\kappa \mathrm{B}$ and leads to nuclear translocation. In the nucleus, $\mathrm{NF}-\kappa \mathrm{B}$ regulates the expression of downstream inflammation factors, such as TNF- $\alpha$ and IL-1 $\beta$, via the p65 subunit, which serves a critical role in kidney IRI $(43,44)$. In the present study, MEF2C and KLF2 expression levels were significantly increased, whereas NF- $\kappa \mathrm{B}$ p 65 , IL-1 $\beta$ and TNF- $\alpha$ expression levels were significantly decreased in HMP livers compared with CS livers. The results suggested that KLF2/NF- $\mathrm{B}$-dependent inflammation was associated with the protective effects of HMP in DCD livers.

However, the differences between HMP and CS at the $1 \mathrm{~h}$ timepoint were not statistically significant, which may be explained by the fact that MEF2C and KLF2 are exclusively induced by laminar flow and not by disturbed flow $(37,42)$. Based on the present findings it was speculated that during perfusion, the perfusion flow is constant, but the vessels of the liver are flexible. At the beginning of perfusion, intrahepatic resistance may be relatively high due to thrombi, and the perfusion flow may not effectively eliminate toxic metabolites and provide adequate nutrients to the liver, thus inducing MEF2C and KLF2 expression. Therefore, sufficient perfusion times are required, and this requires further investigation. The present study indicated that the expression levels of MEF2C and KLF2 peaked after $3 \mathrm{~h}$ of HMP and then gradually reduced with increased perfusion duration. In addition, the levels of NF- $\kappa \mathrm{B}$ p 65, IL-1 $\beta$, and TNF- $\alpha$ were lowest at $3 \mathrm{~h}$ compared with the other timepoints, which was consistent with the morphological alterations that were observed. A potential explanation for the aforementioned results is that $3 \mathrm{~h}$ of HMP is sufficient to achieve a steady laminar flow, allowing effective liver perfusion and significant induction of MEF2C and KLF2 expression. Nevertheless, no matter how effective HMP may be, it can only delay organ damage caused by cold ischemia (35). With prolonged perfusion times, livers experienced edema, and because hepatic sinuses were not elastic, the intrahepatic resistance increased and the perfusion flow in liver sinuses decreased, which resulted in decreased MEF2C and KLF2 expression. Therefore, the results indicated that the optimal perfusion time for HMP to effectively inhibit inflammation was $3 \mathrm{~h}$.

Based on the aforementioned results, the IPRL system was used to imitate transplantation and evaluate the protective effects of HMP. A significant reduction in ALT, AST and LDH levels was observed in HMP-perfused livers compared with CS-perfused livers at different reperfusion time points.
Moreover, liver oxygen consumption was significantly higher in the HMP group compared with the CS groups. Bile production was also higher in the HMP group compared with the CS group, but the difference was not statistically significant, possibly because the liver was only perfused via the portal vein and not via the hepatic artery. The hepatic artery is the only source of blood supply to the bile duct; therefore, the biliary tract may not have received sufficient oxygen supply in the present study (45). To elucidate the mechanisms underlying the protective effects of HMP, the expression levels of $\mathrm{NF}-\kappa \mathrm{B}$ and eNOS/NO signaling pathway-related proteins were measured. The NF- $\mathrm{NB}$ signaling pathway serves an important role during the inflammatory response $(16,40)$, and eNOS is the most important NOS isoform that constitutively produces NO under physiological conditions (23). In ECs, the homeostasis of eNOS/NO displays an important role in oxidative stress and the process IRI $(25,46)$. The results of the present study indicated that HMP alleviated the inflammatory response and oxidative stress injury during IRI by inducing KLF2 expression, which inhibited NF- $\kappa B$ signaling and activated eNOS/NO signaling.

The present study had a number of limitations. The optimal perfusion time for DCD livers was determined by focusing only on KLF2 levels rather than the total gene expression profile. In addition, the ex vivo perfusion model used in the present study only allowed for a short observation period after reperfusion; therefore, long-term effects, including those of transplantation, were not assessed and require further investigation. Finally, the results were obtained using an ex vivo model that was perfused via the portal vein, so the effects of perfusion via the hepatic artery or both blood vessels were not examined.

The present study further suggested that HMP was more effective at preserving DCD livers compared with CS. The results indicated that the optimal perfusion time for HMP was $3 \mathrm{~h}$, and that shorter or longer perfusion times did not achieve the desired perfusion effect. In addition, the protective effects of HMP were primarily exerted via attenuation of the $\mathrm{KLF} 2 / \mathrm{NF}-\kappa \mathrm{B} / \mathrm{eNOS}$-dependent inflammatory response and oxidative stress.

\section{Acknowledgements}

The authors would like to thank the doctors and students of Zhongnan Hospital of Wuhan University for their help in this research, including Professor Guizhu Peng, Vice-Professor Shaojun Ye, Vice-Professor Dawei Zhou, Dr Zhiping Xia, Mrs. Ling Li, Mrs. Xin Zhou, Miss Ruizeng Luo, Miss Bingru Zhao, Mr. Juntao Liang, Mr. Huikai Zhang and Mr. Wenhao Su.

\section{Funding}

The present study was supported by the National Natural Science Foundation of China (grant no. 81570079) and the National Natural Science Foundation of China-Xinjiang joint fund (grant no. U1403222).

\section{Availability of data and materials}

The datasets used and/or analyzed during the current study are available from the corresponding author on reasonable request. 


\section{Authors' contributions}

$\mathrm{XH}, \mathrm{YW}$ and QY designed the study, performed the experiments, analyzed the data and wrote the manuscript. WW and $\mathrm{CZ}$ performed the experiments, contributed to the design of the study and helped to write the manuscript. $\mathrm{XH}, \mathrm{CZ}$ and $\mathrm{ZZ}$ contributed to the establishment of the liver perfusion system. WH and ZL established the rat model of DCD. WW and ZZ provided guidance and revised the manuscript. YW and QY also provided overall guidance. All authors read and approved the final manuscript.

\section{Ethics approval and consent to participate}

The present study was approved by the Ethical Committee of Wuhan University.

\section{Patient consent for publication}

Not applicable.

\section{Competing interests}

The authors declare that they have no competing interests.

\section{References}

1. Neuberger J: An update on liver transplantation: A critical review. J Autoimmun 66: 51-59, 2016.

2. Dar WA, Sullivan E, Bynon JS, Eltzschig H and Ju C: Ischaemia reperfusion injury in liver transplantation: Cellular and molecular mechanisms. Liver Int. 2019;39(5):788-801.

3. Eltzschig HK, Eckle T. Ischemia and reperfusion - from mechanism to translation. Nat Med 17: 1391-1401, 2011.

4. Zhai Y, Petrowsky H, Hong JC, Busuttil RW and Kupiec-Weglinski JW: Ischaemia-reperfusion injury in liver transplantation - from bench to bedside. Nat Rev Gastroenterol Hepatol 10: 79-89, 2013.

5. Dengu F, Abbas SH, Ebeling G and Nasralla D: Normothermic machine perfusion (NMP) of the liver as a platform for therapeutic interventions during ex-vivo liver preservation: A Review. J Clin Med 9: E1046, 2020.

6. Xue S, He W, Zeng X, Tang Z, Feng S, Zhong Z, Xiong Y, Wang $Y$ and Ye Q: Hypothermic machine perfusion attenuates ischemia/reperfusion injury against rat livers donated after cardiac death by activating the Keap1/Nrf2 ARE signaling pathway. Mol Med Rep 18: 815-826, 2018.

7. Guarrera JV, Henry SD, Samstein B, Reznik E, Musat C, Lukose TI, Ratner LE, Brown RS Jr, Kato T and Emond JC: Hypothermic machine preservation facilitates successful transplantation of 'orphan' extended criteria donor livers. Am J Transplant 15: 161-169, 2015.

8. Guarrera JV, Henry SD, Samstein B, Odeh-Ramadan R, Kinkhabwala M, Goldstein MJ, Ratner LE, Renz JF, Lee HT, Brown RS Jr, et al: Hypothermic machine preservation in human liver transplantation: The first clinical series. Am J Transplant 10: 372-381, 2010.

9. Monbaliu D, Liu Q, Libbrecht L, De Vos R, Vekemans K, Debbaut C, Detry O, Roskams T, van Pelt J and Pirenne J: Preserving the morphology and evaluating the quality of liver grafts by hypothermic machine perfusion: A proof-of-concept study using discarded human livers. Liver Transpl 18: 1495-1507, 2012.

10. Zeng C, Hu X, He W, Wang Y, Li L, Xiong Y and Ye Q: Hypothermic machine perfusion ameliorates inflammation during ischemia reperfusion injury via sirtuin 1 mediated deacetylation of nuclear factor $\kappa \mathrm{B}$ p65 in rat livers donated after circulatory death. Mol Med Rep 16: 8649-8656, 2017.

11. Lüer B, Fox M, Efferz P and Minor T: Adding pulsatile vascular stimulation to venous systemic oxygen persufflation of liver grafts. Artif Organs 38: 404-410, 2014.
12. Doddaballapur A, Michalik KM, Manavski Y, Lucas T, Houtkooper RH, You X, Chen W, Zeiher AM, Potente M, Dimmeler S, et al: Laminar shear stress inhibits endothelial cell metabolism via KLF2-mediated repression of PFKFB3. Arterioscler Thromb Vasc Biol 35: 137-145, 2015.

13. Liu Z, Zhong Z, Lan J, Li M, Wang W, Yang J, Tang C, Wang J, Ye S, Xiong Y, et al: Mechanisms of hypothermic machine perfusion to decrease donation after cardiac death graft inflammation: through the pathway of upregulating expression of KLF2 and inhibiting TGF- $\beta$ signaling. Artif Organs 41: 82-88, 2017.

14. Hide D, Ortega-Ribera M, Garcia-Pagan JC, Peralta C, Bosch J and Gracia-Sancho J: Effects of warm ischemia and reperfusion on the liver microcirculatory phenotype of rats: Underlying mechanisms and pharmacological therapy. Sci Rep 6: 22107, 2016.

15. Nayak L, Lin Z and Jain MK: 'Go with the flow': How Krüppel-like factor 2 regulates the vasoprotective effects of shear stress. Antioxid Redox Signal 15: 1449-1461, 2011.

16. Hayden MS and Ghosh S: Shared principles in NF-kappaB signaling. Cell 132: 344-362, 2008.

17. Marrone G, Maeso-Díaz R, García-Cardena G, Abraldes JG, García-Pagán JC, Bosch J and Gracia-Sancho J: KLF2 exerts antifibrotic and vasoprotective effects in cirrhotic rat livers: Behind the molecular mechanisms of statins. Gut 64: 1434-1443, 2015.

18. Fledderus JO, van Thienen JV, Boon RA, Dekker RJ, Rohlena J, Volger OL, Bijnens AP, Daemen MJ, Kuiper J, van Berkel TJ, et al: Prolonged shear stress and KLF2 suppress constitutive proinflammatory transcription through inhibition of ATF2. Blood 109: 4249-4257, 2007.

19. Boon RA, Fledderus JO, Volger OL, van Wanrooij EJ, Pardali E, Weesie F, Kuiper J, Pannekoek H, ten Dijke P and Horrevoets AJ: KLF2 suppresses TGF-beta signaling in endothelium through induction of Smad7 and inhibition of AP-1. Arterioscler Thromb Vasc Biol 27: 532-539, 2007.

20. Das H, Kumar A, Lin Z, Patino WD, Hwang PM, Feinberg MW, Majumder PK and Jain MK: Kruppel-like factor 2 (KLF2) regulates proinflammatory activation of monocytes. Proc Natl Acad Sci USA 103: 6653-6658, 2006.

21. Wang W, Ha CH, Jhun BS, Wong C, Jain MK and Jin ZG: Fluid shear stress stimulates phosphorylation-dependent nuclear export of HDAC5 and mediates expression of KLF2 and eNOS. Blood 115: 2971-2979, 2010.

22. Chu H, Li H, Guan X, Yan H, Zhang X, Cui X, Li X and Cheng M: Resveratrol protects late endothelial progenitor cells from TNF- $\alpha$-induced inflammatory damage by upregulating Krüppel-like factor-2. Mol Med Rep 17: 5708-5715, 2018.

23. Erkens R, Suvorava T, Kramer CM, Diederich LD, Kelm M and Cortese-Krott MM: Modulation of Local and Systemic Heterocellular Communication by Mechanical Forces: A Role of Endothelial Nitric Oxide Synthase. Antioxid Redox Signal 26: 917-935, 2017.

24. Fleming I: Molecular mechanisms underlying the activation of eNOS. Pflugers Arch 459: 793-806, 2010.

25. Liu Z, Zhang X, Xiao Q, Ye S, Lai CH, Luo J, Huang X, Wang W, Zeng C, Zhong Z, et al: Pretreatment Donors after Circulatory Death with Simvastatin Alleviates Liver Ischemia Reperfusion Injury through a KLF2-Dependent Mechanism in Rat. Oxid Med Cell Longev 2017: 3861914, 2017.

26. National Research Council (US) Committee for the Update of the Guide for the Care and Use of Laboratory Animals: Guide for the Care and Use of Laboratory Animals, 8th edition. National Academies Press (US), Washington, DC, 2011.

27. Schlegel A, Kron P, Graf R, Dutkowski P and Clavien PA: Warm vs. cold perfusion techniques to rescue rodent liver grafts. J Hepatol 61: 1267-1275, 2014.

28. Kamada $\mathrm{N}$ and Calne RY: A surgical experience with five hundred thirty liver transplants in the rat. Surgery 93: 64-69, 1983.

29. Minor T, Manekeller S, Sioutis M and Dombrowski F: Endoplasmic and vascular surface activation during organ preservation: Refining upon the benefits of machine perfusion. Am J Transplant 6: 1355-1366, 2006.

30. Pizarro MD, Rodriguez JV, Mamprin ME, Fuller BJ, Mann BE, Motterlini R and Guibert EE: Protective effects of a carbon monoxide-releasing molecule (CORM-3) during hepatic cold preservation. Cryobiology 58: 248-255, 2009.

31. Balaban CL, Rodriguez JV and Guibert EE: Delivery of the bioactive gas hydrogen sulfide during cold preservation of rat liver: Effects on hepatic function in an ex vivo model. Artif Organs 35: 508-515, 2011. 
32. Suzuki S, Toledo-Pereyra LH, Rodriguez FJ and Cejalvo D: Neutrophil infiltration as an important factor in liver ischemia and reperfusion injury. Modulating effects of FK506 and cyclosporine. Transplantation 55: 1265-1272, 1993.

33. Jha $\mathrm{P}$ and Das H: KLF2 in regulation of $\mathrm{NF}-\kappa \mathrm{B}$-mediated immune cell function and inflammation. Int J Mol Sci 18: E2383, 2017.

34. Weissenbacher A, Vrakas G, Nasralla D and Ceresa CDL: The future of organ perfusion and re-conditioning. Transpl Int 32: $586-597,2019$.

35. Schlegel A and Dutkowski P: Role of hypothermic machine perfusion in liver transplantation. Transpl Int 28: 677-689, 2015.

36. Op den Dries S, Sutton ME, Karimian N, de Boer MT, Wiersema-Buist J, Gouw AS, Leuvenink HG, Lisman T and Porte RJ: Hypothermic oxygenated machine perfusion prevents arteriolonecrosis of the peribiliary plexus in pig livers donated after circulatory death. PLoS One 9: e88521, 2014.

37. Niu N, Xu S, Xu Y, Little PJ and Jin ZG: Targeting Mechanosensitive Transcription Factors in Atherosclerosis. Trends Pharmacol Sci 40: 253-266, 2019.

38. McKinsey TA, Zhang CL and Olson EN: MEF2: A calcium-dependent regulator of cell division, differentiation and death. Trends Biochem Sci 27: 40-47, 2002.

39. Potthoff MJ and Olson EN: MEF2: A central regulator of diverse developmental programs. Development 134: 4131-4140, 2007.

40. Xu Z, Yoshida T, Wu L, Maiti D, Cebotaru L and Duh EJ: Transcription factor MEF2C suppresses endothelial cell inflammation via regulation of NF- $\mathrm{BB}$ and KLF2. J Cell Physiol 230: 1310-1320, 2015

41. Lu YW, Lowery AM, Sun LY, Singer HA, Dai G, Adam AP, Vincent PA and Schwarz JJ: Endothelial Myocyte Enhancer Factor 2c Inhibits Migration of Smooth Muscle Cells Through Fenestrations in the Internal Elastic Lamina. Arterioscler Thromb Vasc Biol 37: 1380-1390, 2017.
42. Parmar KM, Nambudiri V, Dai G, Larman HB, Gimbrone MA Jr and García-Cardeña G: Statins exert endothelial atheroprotective effects via the KLF2 transcription factor. J Biol Chem 280: 26714-26719, 2005.

43. Li Z, Nickkholgh A, Yi X, Bruns H, Gross ML, Hoffmann K, Mohr E, Zorn M, Büchler MW and Schemmer P: Melatonin protects kidney grafts from ischemia/reperfusion injury through inhibition of NF-kB and apoptosis after experimental kidney transplantation. J Pineal Res 46: 365-372, 2009.

44. Zhang J, Xia J, Zhang Y, Xiao F, Wang J, Gao H, Liu Y, Rong S, Yao Y, Xu G, et al: HMGB1-TLR4 signaling participates in renal ischemia reperfusion injury and could be attenuated by dexamethasone-mediated inhibition of the ERK/NF- $\mathrm{BB}$ pathway. Am J Transl Res 8: 4054-4067, 2016.

45. Carnevale ME, Balaban CL, Guibert EE, Bottai $H$ and Rodriguez JV: Hypothermic machine perfusion versus cold storage in the rescuing of livers from non-heart-beating donor rats. Artif Organs 37: 985-991, 2013

46. Ajamieh H, Farrell GC, McCuskey RS, Yu J, Chu E, Wong HJ, Lam W and Teoh NC: Acute atorvastatin is hepatoprotective against ischaemia-reperfusion injury in mice by modulating eNOS and microparticle formation. Liver Int 35: 2174-2186, 2015.

This work is licensed under a Creative Commons Attribution-NonCommercial-NoDerivatives 4.0 International (CC BY-NC-ND 4.0) License. 